\title{
Mid- and Far-Infrared Study of X-ray-emitting Dusty Elliptical Galaxies
}

\author{
H. Kaneda ${ }^{1}$, T. Onaka ${ }^{2}$, T. Kitayama ${ }^{3}$, Y. Okada ${ }^{1}$, and I. Sakon ${ }^{2}$ \\ ${ }^{1}$ Institute of Space and Astronautical Science, Sagamihara, Kanagawa 229-8510, Japan \\ email: kaneda@ir.isas.jaxa.jp \\ ${ }^{2}$ Department of Astronomy, University of Tokyo, Bunkyo-ku, Tokyo 113-0003, Japan \\ ${ }^{3}$ Department of Physics, Toho University, Funabashi, Chiba 274-8510, Japan
}

\begin{abstract}
We study nearby X-ray-emitting dusty elliptical galaxies in mid- and far-infrared by using the Spitzer Space Telescope and AKARI, the Japanese infrared astronomical satellite.
\end{abstract}

Keywords. ISM: dust, extinction — galaxies: elliptical and lenticular, cD — galaxies: ISM

Elliptical galaxies provide dust with a unique environment, i.e. old stellar radiation fields and interstellar media (ISM) dominated by hot plasma. The presence of a considerable amount of interstellar dust in elliptical galaxies is quite common; some galaxies show even the presence of polycyclic aromatic hydrocarbons (PAHs) and very small grains (VSGs). How can such small dust particles as PAHs and VSGs survive in the plasma environment? To discuss the origins and fates of the dust, we study nearby X-ray-emitting dusty elliptical galaxies in mid- and far-infrared with Spitzer (GO1\&GO3) and AKARI. We have succeeded in the detection of PAH emission features (Kaneda et al. 2005); the spectra show unusually strong features at wavelengths of $>10 \mu \mathrm{m}$ relative to those at $<10 \mu \mathrm{m}$, probably reflecting the peculiar ISM conditions (Fig. 1). We have found some signatures of interaction of interstellar dust with hot plasma. Following Spitzer, AKARI was launched successfully in February, 2006 (Fig. 2); their combination would greatly improve our understanding of the evolutionary history of dust in elliptical galaxies.

This work is based on observations with Spitzer operated by the JPL, CIT under NASA contract, and $A K A R I$, a JAXA project with the participation of ESA.

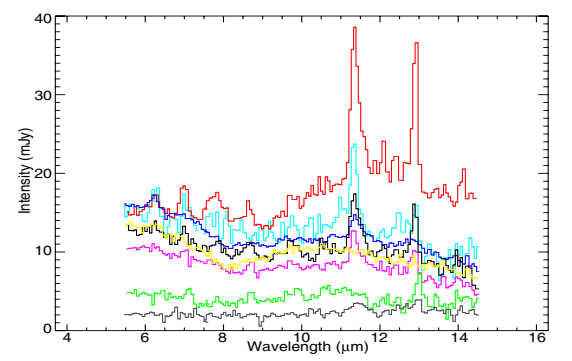

Figure 1. Mid-infrared spectra of our sample galaxies obtained with the Spitzer IRS/SL.
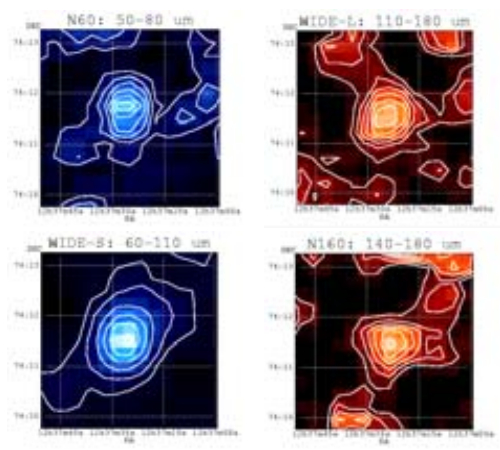

Figure 2. Far-infrared 4-band images of NGC4589 obtained with the AKARI FIS.

\section{Reference}

Kaneda, H., Onaka, T., \& Sakon, I. 2005, ApJ, 632, L83. 